ORIGINAL ARTICLE

\title{
Families' views on ward rounds in neonatal units
}

\author{
R Bramwell, M Weindling for the FVWR Research Team
}

Arch Dis Child Fetal Neonatal Ed 2005;90:F429-F431. doi: 10.1136/adc.2004.061168

See end of article for
authors' affiliations
......................
Correspondence to:
Dr Bramwell, Department
of Clinical Psychology,
University of Liverpool,
Whelan Building,
Brownlow Hill, Liverpool
L69 3GB, UK'
rosb@liv.ac.uk
Accepted
2 December 2004

See end of article for authors' affiliations

Correspondence to:

Dr Bramwell, Department

University of Liverpool,

Whelan Building,

Brownlow Hill, Liverpool

L69 3GB, UK

2 December 2004

\begin{abstract}
Objective: To discover parental preferences about visiting during ward rounds.
Design: Survey using a short structured interview

Setting and participants: Families of babies cared for in a regional neonatal intensive care unit.

Results: Eighty six respondents, no refusals. Sixty three had visited during a ward round, and 13 had come in especially for the round. About half had overheard conversations about other babies or thought discussions about their baby had been overheard. Concerns about these experiences were only expressed by respondents who had actually experienced overhearing. Parents and families had little information about the ward round, held diverse views, and expressed different priorities. They described a mixture of concerns about communication, practicalities, and issues of ethics and principle. Confidentiality was a matter of concern for some, but many parents expected some sharing of information between families on the unit.

Conclusions: Units should consider: the information they have for parents about ward rounds; the possibility that consultations may be overheard; the opportunities for parents to communicate with the clinical team.
\end{abstract}

$\mathrm{T}$ he admission of a baby to the neonatal unit is likely to be a source of great anxiety to parents. ${ }^{1}$ In an attempt to minimise such anxiety, Liverpool Women's Hospital neonatal unit operates a policy of unrestricted visiting. There is evidence that this policy (common in the United Kingdom although not other parts of Europe ${ }^{23}$ ) helps parents and is welcomed by them. ${ }^{4}$

The ward round (WR) is central to the care of the patient. At Liverpool Women's Hospital, parents are allowed to remain during WRs, a policy considered to foster openness and to avoid parents feeling excluded from their baby's care. Literature shows that parents are keen to be fully informed by medical staff, and the WR may be a good opportunity (but not the only one) for parents to meet the medical team. ${ }^{6}$ However, a paediatric study suggested frequent breaches of confidentiality during WRs, ${ }^{8}$ and some hospitals have taken the view that it is stressful for parents to be present at such a time, and that their presence may inhibit discussion between professionals. Furthermore, it is sometimes argued that parents may be unable to follow the detail of such complex discussions, may be inhibited by the presence of so many people, are unlikely to want to take part, and may overhear discussions that relate to other sick babies. Similar problems have been found in studies of adult WRs. ${ }^{9}$

This study was undertaken to discover parental preferences and to inform a policy on visiting during the WR. It comprised a survey to discover present practice and a short structured interview.

\section{METHOD \\ Telephone survey of policies across neonatal units in the United Kingdom}

Thirty seven tertiary prenatal centres in the United Kingdom identified in a previous published study ${ }^{10}$ were telephoned. The researcher asked to speak to the unit manager or senior nurse on duty, and encouraged respondents to consult colleagues.

\section{Families' views}

Design

The design was a short structured interview.

\section{Setting}

The neonatal unit at Liverpool Women's Hospital has 50 cots: 15 are intensive care, 20 special care, and 15 high dependency. Babies receiving intensive and high dependency care are in six rooms with five cots in each; babies receiving special care are in a single large room. Cots are fairly close together, generally with room for about two people between each. Some babies are very seriously ill, and there are, on average, one or two deaths each week. A consultant led WR takes place each morning between 0830 and 1030. It involves a group of people standing around the baby. All are qualified staff; students only attend occasionally. There are usually between eight and ten people. They comprise the junior medical staff who looked after the baby overnight (a registrar and senior house officer), the doctors who will take over the baby's care during the day (two registrars and two senior house officers), between one and three advanced neonatal nurse practitioners, and the nurse who is caring for the baby. There may be others too-for example, a social worker, the nursing shift leader, other medical specialists.

\section{Procedure}

Neonatal nurses first approached families. All participants gave signed consent.

Parents were interviewed individually. Partners other than genetic parents and grandparents were also eligible for inclusion. A structured interview included both closed questions and an invitation for further open comments on all questions. Responses were noted on paper.

This study was approved by the Liverpool Paediatric Local Research Ethics Committee.

\section{Analysis}

Responses to the closed questions were analysed using SPSS for Windows. Participants' open comments were subjected to a thematic content analysis. ${ }^{11}$

\section{RESULTS}

Survey of policies across neonatal units in the United Kingdom

Thirty four of the 37 centres responded. They comprised between five and 16 cots (median 10), and had one 
$(\mathrm{n}=18)$, two $(\mathrm{n}=13)$, or three $(\mathrm{n}=3)$ WRs daily. All responding units had an open access visiting policy. Eight allowed all parents to be present during WRs, eight did not allow any parents to be present, and 18 allowed only parents of the baby being discussed to be present.

\section{Families' views}

Respondents

Eighty six family members responded, with no refusals. Respondents comprised 59 (69\%) mothers, 24 (28\%) fathers, and three $(4 \%)$ grandparents. The babies they were visiting had been on the unit between 1 and 167 days (median 14).

\section{Experience of WRs}

Sixty three $(73 \%)$ respondents remembered having visited during a WR, of whom 13 (15\% of sample) had come in especially to talk to the doctors during the WR. Some problems were caused by a lack of information about the timing and nature of WRs. Some parents would have liked to have come in for the WR but did not know when it was. Another, not knowing a WR would be in progress, said, "I was horrified to find so many people around my baby, I thought something was seriously wrong."

Some respondents considered WRs a good source of information: "It's good to hear what they are saying and doing about the baby's condition". They appreciated the opportunity to be there for WRs, especially when there was a specific problem or when they felt anxious about their infant's progress. When families were asked how they would feel about being present when a group of doctors were discussing their baby's progress, $74(86 \%)$ indicated that "It would be good to hear what the doctors had to say". However, six (7\%) felt that they would find it difficult to talk with a whole group of doctors, and some specifically said they found the WR "not enjoyable" or would avoid it. Several commented that the WR might be intimidating or excluding for others-for example, "It must be hard for less confident parents".

The quality of interactions with health professionals was an important element of the families' responses to WRs. Some reported positive experiences, with comments like, "Doctors included me in the conversation". Others felt that doctors did not include them and one parent said it would be good "If all the doctors could make an effort to talk to the parents". Some respondents perceived a change in staff behaviour when families were present. The number of staff attending the wards was also found to be intimidating. Medical jargon was perceived as a barrier to understanding.

\section{Concerns about confidentiality}

Over half the respondents thought other parents had overheard what was being said about their baby (table 1). Of these, $11(20 \%)$ were definitely or slightly bothered by this. Of those who had not had this experience, all stated that they did not think that this would bother them.

Over half the respondents had overheard what doctors were saying about another baby on the unit (table 1). Of these, $10(16 \%)$ were worried or embarrassed by the experience. Of those who had not had the experience of being overheard, none believed they would feel worried or embarrassed in this situation.

The qualitative data gave further insight. Parents commented that overhearing was inevitable because of the small size of the nurseries. Some stated that they tried not to listen: they were "Just there to concentrate on their own child". Other parents said that they "Don't take any notice" about what is being said about another baby's condition as "Everyone is focusing all their energy on their own baby". Others felt that "We are there for a common reason, our children are all sick", and that there was "Talking between parents anyway". One participant reported a positive side: "Overhearing success of other infants can be very encouraging."

A few specifically identified overhearing such conversations as a breach of confidentiality. For instance, one participant reported feelings of anxiety and distress when she overheard a discussion about a baby and that infant's parents were not present. Others said, "Some things just families should hear", and "If the matter was serious then it shouldn't be discussed openly in a ward round".

\section{Views on ward opening arrangements}

When participants were asked what it would mean to them and their family if they could not come in to visit during WRs, $28(33 \%)$ said it would make it very difficult or impossible for them to visit their baby. All respondents except for one were satisfied or very satisfied with current WR arrangements. The only dissatisfaction expressed with current arrangements was by a mother who found it embarrassing to breast feed at WR times. A major concern was that feeding times may be missed if the unit was not open all day, "I haven't seen my baby overnight so I want to be there for the early morning feed".

The WR was held relatively early in the day so that, as one respondent remarked, there are "Not normally people here". However, whereas some respondents simply commented that they liked the open access, some were against closing the unit for WRs on grounds of practicality and principle: "It would be very difficult to visit as my husband has to drop me off at 8 am before ... work". However, some respondents were in favour of closure for WRs: "The doctors have to do their job". Some suggested that visitors other than parents should be excluded during WRs.

\section{DISCUSSION}

This study opens a debate on clinical practice by using the paradigm of the morning, consultant led WR on a neonatal intensive care unit. The primary purpose of the WR is to enable detailed discussion by professionals of a patient's condition and care. Some parents knew very little about the purpose, nature, and timing of WRs. In the absence of such information, parents may draw on "lay" representations of hospital life, perhaps including soap operas and comedies. Even the excellent BLISS parent information guide ${ }^{12}$ includes

Table 1 Experiences of overhearing

\begin{tabular}{llll}
\hline Question & Response & Number & $\%$ \\
\hline Have you ever thought that other parents could overhear & No, never & 32 & 37 \\
what was being said about your baby by the doctors? & Yes, just once & 8 & 9 \\
& Yes, several times & 46 & 54 \\
Have you ever noticed that you could overhear what other & No, never & 24 & 28 \\
doctors were saying about another baby on the unit? & Yes, just once & 10 & 12 \\
& Yes, several times & 52 & 61 \\
\hline
\end{tabular}




\section{What is already known on this topic}

Issues of privacy and confidentiality have been highlighted in previous studies of adult and paediatric ward rounds, but there is a lack of research into families' views of ward rounds on a neonatal unit

just four sentences under the heading "Doctor's round", where parents are told that "During the morning round the staff will plan your baby's care". The number and type of staff involved, the amount of time spent with each baby, and the likely nature of the discussion are not described.

This study has a number of strengths and weaknesses. It was carried out on a single unit, and there is clearly scope for further research to compare experiences in units with different policies. All families approached were prepared to participate, facilitated by the use of a short structured interview, which meant that there was no literacy barrier. This approach, where open and closed questions were combined, gave further insight into the quantitative data, and allowed some new themes, not covered by the closed questions, to emerge. Where open responses were offered, it could not be assumed that other respondents would not have made a similar point.

Comments about how "other parents" might feel may be seen as speculation, but may also reflect a communication strategy whereby parents could voice their own concerns without having to admit to negative feelings or criticism of staff. Parents expressed very different, sometimes almost opposing, viewpoints. The parents themselves were, of course, a diverse set of people who had varying experiences. Such diversity needs to be borne in mind in considering best practice for the conduct of WRs.

Although the paradigm of the consultant led WR was used, there are other situations on all hospital wards where discussions take place between professionals, including nursing handovers.

An important benefit for parents in attending WRs was the opportunity to be informed about their baby's condition and talk to the medical staff. However, it is questionable whether parents' communication needs are best met within the structure of the WR. Although parents may see the WR as a source of information, this is not its primary purpose. For instance, health professionals often use specialist language and the presence of a large number of health professionals may be intimidating. Other research in neonatal units has also recognised that communication can be problematic. ${ }^{13-15}$ Meeting members of the team on a separate occasion may be more effective.

For some parents, being able to visit the ward at all times was a matter of principle, but patient confidentiality may present a competing imperative. Overhearing or being overheard was only a problem for parents who had had that experience, and then only for some. This suggests the need to focus research on confidentiality issues on those who have experienced breaches. It was suggested that overhearing was most intrusive when the issues were "serious".

Alternatives to the traditional WR include: asking parents to leave the room during the WR, but talking to them separately; conducting the WR in a separate room; asking parents to leave the room but asking them back in when their child is being seen, although this may be awkward and make a long WR even longer.
What this study adds

- Current practice on visiting during ward rounds varies between neonatal units in the United Kingdom

- Overhearing and being overheard are relatively common experiences

- Overhearing and being overheard can cause concern for those who have had this experience

- Families lack basic information about the ward round.

- Families see ward rounds as an opportunity to obtain information, but many are intimidated by them

- There is no simple consensus among families on what the policy on access during ward rounds should be

The lack of information about WRs, identified by this study, can be addressed by posters and information leaflets. Clinical practice needs to take into account the observation that overhearing and being overheard are distressing experiences for some. Parents need to be able to communicate with the clinical team, but this is probably not best done during a WR.

\section{Authors' affiliations}

R Bramwell, Department of Clinical Psychology, University of Liverpool, Liverpool L69 3GB, UK

M Weindling, School of Reproductive and Developmental Medicine, University of Liverpool, Neonatal Unit, Liverpool Women's Hospital, Liverpool L8 7SS, UK

Competing interests: none declared

The Families' Views on Ward Rounds (FVWR) research team comprised Rosie Barnes, Jill Dixon, Vicky Priest, Ruth Taylor, and Rachel Williams who conducted the interviews reported in this paper.

\section{REFERENCES}

1 Padden T, Glenn S. Maternal experiences of preterm birth and neonatal intensive care. J Reprod Infant Psychol 1997;15:121-39.

2 Reid M, Andersen E, and EC Study Group of Parental Involvement in Neonatal Care. Variations in family visiting policies in neonatal intensive care units in eleven EC countries. Paediatr Perinat Epidemiol 1994;8:41-52.

3 Cuttini M, Rebagliato M, Bortoli P, et al. Parental visiting, communication, and participation in ethical decisions: a comparison of neonatal policies in Europe. Arch Dis Child Fetal Neonatal Ed 1999:81:F84-91.

4 Yu VYH, Jamieson J, Astbury J. Parents' reactions to unrestricted parental contact with infants in intensive care nursery. Med J Aust $1981 ; 1: 294-6$.

5 Perlman NB, Freedman JL, Abramovitch R, et al. Informational needs of parents of sick neonates. Pediatrics 1991;88:512-18.

6 Birtwistle L, Houghton JM, Rostill H. A review of a surgical ward round in a large paediatric hospital: does it achieve its aims? Med Educ 2000;34:398-403.

7 Shellabarger SG, Thompson TL. The critical times: meeting parental communication needs throughout the NICU experience. Neonatal Netw 1993; 12:39-45.

8 Rylance G. Privacy, dignity, and confidentiality: interview study with structured questionnaire. BMJ 1999;318:301.

9 Steele SJ, Morton DJB. The ward round. Lancet 1978;2:85-6.

10 Parmanum J, Field D, Rennie J, et al. National census of availability of neonatal intensive care. BMJ 2000:321:727-79.

11 Richardson JTE. Handbook of qualitative research methods. Leicester: BPS Books, 1996

12 Moore P. BLISS parent information guide. London: BLISS, 2004.

13 Redshaw ME, Harris A. Maternal perceptions of neonatal care. Acta Paediatr 1995;84:593-8.

14 Wereszak J, Miles MS, Holditch-Davis D. Maternal recall of the neonatal intensive care unit. Neonatal Netw 1997; 16:33-9.

15 Affleck G, Tennen H, Rowe J. Mothers' remembrances of newborn intensive care: a predictive study. J Pediatr Psychol 1990;15:67-81. 